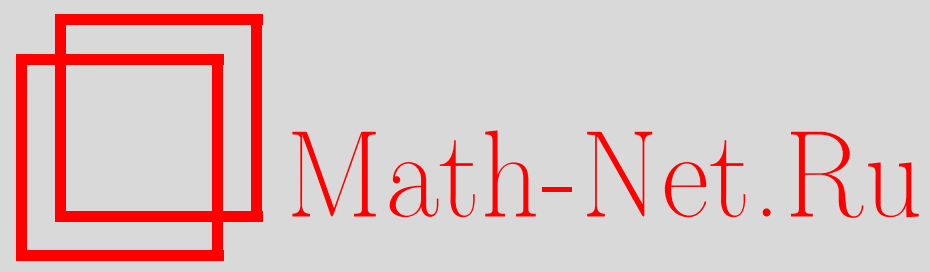

П. В. Ролдугин, О числе максимально негамильтоновых графов, Матем. заметки, 2004, том 75, выпуск 5, 702-710

DOI: https://doi.org/10.4213/mzm66

Использование Общероссийского математического портала Math-Net.Ru подразумевает, что вы прочитали и согласны с пользовательским соглашением http://www. mathnet.ru/rus/agreement

Параметры загрузки:

IP: 54.198 .67 .100

26 апреля 2023 г., 10:54:31 


\title{
О ЧИСЛЕ МАКСИМАЛЬНО НЕГАМИЛЬТОНОВЫХ ГРАФОВ
}

\section{П. В. Ролдугин}

\begin{abstract}
В данной работе изучаются максимально негамильтоновые графы (МНГ графы), т.е. негамильтоновы граффы, в которые нельзя добавить новое ребро без нарушения его негамильтоновости. Показано, что изучение МНГ графов можно свести к изучению так называемых упрощенных МНГ графов. Получены ограничения на структуру максимальных клик упрощенных МНГ графов, оценены порядки и число таких графов.

Библиография: 5 названий.
\end{abstract}

0. Введение. В этой работе мы рассматриваем только непомеченные конечные неориентированные графы без петель и кратных ребер. Термины, относящиеся к теории графов, можно найти в учебнике [1]. Напомним, что гамильтониановым графом называется граф, в котором существует простой цикл, проходящий по всем вершинам графа (гамильтонов цикл). Графы, в которых такой цикл отсутствует, назьваются негамильтоновыми.

В данной работе изучаются так называемые максимально негамильтоновые графы.

ОПРЕДЕЛЕНИЕ 1. Граф $G$ будем называть максимально негамильтоновым графом (сокращенно МНГ графом), если выполнены два условия:

1) графф $G$ негамильтонов;

2) при добавлении хотя бы одного ребра, инцидентного любой паре несмежных вершин, граф $G$ становится гамильтоновым.

Результаты, относящиеся к таким графам, кроме работы автора [2] можно найти такжев работе [3]. Кроме того, понятие МНГ графа возникает при доказательстве теоремы Поша (например, в монографии [4]).

Поскольку любой негамильтонов граф содержится в качестве остовного подграфа в некотором максимально негамильтоновом графе, изучение максимально негамильтоновых графов может позволить продвинуться в вопросах, относящихся к гамильтоновьг циклам, таких, как: построение критериев существования гамильтонова цикла, подсчет числа гамильтоновых (или негамильтоновых) графов и так далее.

В утверждении 1 настоящей работы показано, что изучение МНГ графов можно свести к изучению так называемых упрощенных МНГ графов. В упрощенном графе каждая максимальная клика содержит не более одной собственной вершины, т.е. вершины, принадлежащей этой максимальной клике и не лежащей в других максимальных кликах 
(под максимальной кликой графа $G$ понимается полньй подграф в $G$, не принадлежащий целиком никакому другому полному подграфу графа $G)$.

Пусть $K_{1}, \ldots, K_{r}$ - такая совокупность максимальных клик графа $G$, что каждое ребро графа принадлежит одной из этих клик (в этом случае будем говорить, что объединение указанных клик дает весь граф). Обозначим $R=\{1,2, \ldots, r\}$. Пусть $I-$ подмножество $R$. Обозначим $n_{I}-$ количество вершин, лежащих в каждой из максимальных клик $K_{i}$ для $i \in I$ и не принадлежащих максимальным кликам с номерами из $R \backslash I$. Доказывается (утверждение 3 ), что набор $n_{I}, I \subseteq R$, задаваемьй упрошенньм МНГ графом с $r$ максимальньми кликами, обязан быть решением некоторой системы линейных неравенств (так назьваемой МНГ-системы), задаваемой значением $r$.

Кроме того, получены верхние оценки порядков и числа (утверждение 4 и следствие к нему) упрощенных МНГ графов, которые могут быть представлены в виде объединения $r$ своих максимальных клик; в частности, доказывается, что число таких графов конечно.

Автор выражает благодарность $\Phi$. М. Малышеву за сделанные замечания и внимание к работе.

1. Упрощенные МНГ графы. Собственной вершиной максимальной клики $K$ графа $G$ назовем вершину, принадлежащую клике $K$, и не принадлежащую другим максимальньм кликам графа $G$.

УТВЕРЖДЕНИЕ 1. Пусть $K$ - максимальная клика графа $G$, имеющая собственную вершину. Добавим в граф $G$ вершину а и ребра, соединяющие вершину $а$ и каждую из вершин клики $K$. Полученный такой прочедурой граф $H$ является МНГ графом тогда и только тогда, когда $G$ - МНГ граф.

ДокАЗАТЕЛЬСТво. Пусть $b$ - собственная вершина клики $K$ графа $G$. Предположим, что $G$ - МНГ граф. Докажем, что граф $H$ негамильтонов. Действительно, если граф $H$ гамильтонов, то существует гамильтонов цикл $\bar{t}$, которьй содержит отрезок $k a k^{\prime}$, где $k, k^{\prime}$ - вершины клики $K$. Поскольку ребро $\left(k, k^{\prime}\right)$ принадлежит графу $G$, то, заменяя в цикле $\bar{t}$ отрезок $k a k^{\prime}$ на отрезок $k k^{\prime}$, получаем гамильтонов цикл в графе $G$, что противоречит его негамильтоновости.

Далее, пусть $x, y$ - две несмежные вершины графа $G$. Покажем, что в графе $H$ их соединяет гамильтонова цепь. Существует гамильтонова цепь $x \bar{t} y$ в графе $G$. Поскольку собственная вершина клики смежна только с другими вершинами той же клики, любая гамильтонова цепь должна содержать отрезок $b b^{\prime}$, где $b^{\prime}$ - вершина клики $K$, отличная от $b$. Заменяя этот отрезок отрезком $b a b^{\prime}$, получаем требуемую цеп в графе $H$. Остается доказать, что существует гамильтонова цепь в графе $H$ между вершинами $x$ и $a$, где $x$ - вершина графа $G$, не лежащая в клике $K$. Пусть $y$ - любая вершина клики $K$ графа $G$, не смежная с $x$ (такая вершина найдется, поскольку $x$ не лежит в $K$ ). Тогда в $G$ существует гамильтонова цепь $x \bar{t} y$, соединяющая $x$ и $y$. Цепь $x \bar{t} y a$ есть искомая гамильтонова цепь в графе $H$ между вершинами $x$ и $a$. Следовательно, в графе $H$ между любыми двумя несмежньми вершинами существует гамильтонова цепь и граф $H$ негамильтонов. Это означает, что $H-$ МНГ граф.

Пусть теперь $H$ - МНГ граф. Предположим, что граф $G$ гамильтонов. Но тогда в нем существует гамильтонов цикл, и этот цикл содержит отрезок $b_{1} b b_{2}$, где $b_{1}, b_{2}-$ различные вершины клики $K$, отличные от $b$ (поскольку вершина $b$ - собственная вершина 
клики $K)$. Заменяя в этом гамильтоновом цикле отрезок $b_{1} b b_{2}$ отрезком $b_{1} b a b_{2}$, получаем гамильтонов цикл в графе $H$. Следовательно, граф $G$ негамильтонов.

Пусть $x$ и $y$ - две несмежные вершины графа $G$. Тогда эти вершины не смежны и в графе $H$. Следовательно, в $H$ вершины $x$ и $y$ соединяет гамильтонова цепь. Поскольку $a$ смежна только с вершинами клики $K$ графа $G$, указанная гамильтонова цепь обязана содержать отрезок $b_{1} a b_{2}$, где $b_{1}, b_{2}$ - различные вершины клики $K$. Заменим этот отрезок цепью $b_{1} b_{2}$ и получим гамильтонову цепь в графе $G$, соединяющую вершины $x$ и $y$. Таким образом, $G$ - МНГ граф.

Утверждение 1 доказано.

ОПРЕДЕЛЕниЕ 2. Граф, в котором каждая максимальная клика содержит не более одной собственной вершины, назовем упрощенным графом.

Из утверждения 1 следует, что любой МНГ граф может быть получен последовательным добавлением новых собственных вершин в максимальные клики с собственными вершинами некоторого упрощенного МНГ графа. То есть множество всех МНГ графов разбивается на непересекающиеся семейства, каждое из которых задается упрощенным МНГ графом. Переход от изучения всех МНГ графов к изучению упрощенных МНГ графов имеет принципиальное значение: например, далее показано, что количество упрощенных МНГ графов с фиксированным числом $r$ максимальных клик конечно и получена зависящая от $r$ верхняя оценка их числа.

2. Построение МНГ-системы. Пусть $K_{1}, \ldots, K_{r}$ - совокупность таких максимальных клик графа $G$, что каждое ребро графа принадлежит одной из этих клик. В частности, в качестве $K_{1}, \ldots, K_{r}$ можно взять совокупность всех максимальных клик графа $G$. Обозначим $R=\{1,2, \ldots, r\}$. Пусть $I$ - подмножество $R$. Определим подмножество $M_{I}$ множества вершин графа $G$ следуюшим образом:

$$
M_{I}=\left(\bigcap_{i \in I} K_{i}\right) \backslash\left(\bigcap_{j \in R \backslash I} K_{j}\right) .
$$

Очевидно, что множества $M_{I}$ и $M_{J}$ не пересекаются при различных $I$ и $J$, a $\bigcup_{I \subseteq R} M_{I}$ исчерпывает все множество вершин графа $G$ и $M_{\varnothing}=\varnothing$. Далее нам понадобится следующая лемма.

Лемма 1. Пусть $I$ - произвольное подмножсество в $R$. Тогда любая вериина $а$ из мнохества $M_{I}$ смежсна со всеми вершинами максимальных клик $K_{i}, i \in I$, отличными от а, и только с ними.

ДокАЗАТЕЛЬСТво. Пусть для некоторого $i \in I$ вершина $b$ лежит в максимальной клике $K_{i}$. Тогда, поскольку по определению множества $M_{I}$ вершина $a$ обязана, в частности, лежать в клике $K_{i}$, вершины $a$ и $b$ смежны. Пусть теперь вершина $b$ смежна с вершиной $a$. Найдется максимальная клика $K_{i}$, содержащая ребро $(a, b)$ (возможна такая найдется не одна - возьмем любую из них). В частности, клика $K_{i}$ будет содержать вершину $a$, из-за чего $i \in I$. Если $i$ не принадлежит $I$, то $a$ принадлежит множеству $\left(\bigcup_{j \in R \backslash I} K_{j}\right)$ и, следовательно, не может лежать во множестве $M_{I}$. Лемма 1 доказана.

Обозначим $n_{I}=\left|M_{I}\right|$, где $I$ - произвольное подмножество в $R$. Для МНГ графов множества $M_{I}, I \subseteq R$, содержат относительно небольшое число элементов, а именно справедлива следующая теорема. 
ТЕОрема 1. Пусть $G-$ МНГ граф. Тогда, если $I_{1} \subsetneq I_{2} \subsetneq \cdots \subsetneq I_{t} \subseteq R-$ иепочка вложенных различных подмножеств $R$, причем $\left|I_{1}\right| \geqslant 2$, то

$$
\sum_{i=1}^{t} n_{I_{i}} \leqslant\left|I_{t}\right|-1
$$

ДокАЗАТЕЛЬСТво. Пусть $\bigcup_{i=1}^{t} M_{I_{i}}=\left\{a_{1}, \ldots, a_{m}\right\}$ и $I_{i}=\left\{i_{1}, \ldots, i_{s}\right\}$. Поскольку множества $M_{I}$ и $M_{J}$ не пересекаются при $I \neq J$, то

$$
\sum_{i=1}^{t} n_{I_{i}}=\sum_{i=1}^{t}\left|M_{I_{i}}\right|=\left|\bigcup_{i=1}^{t} M_{I_{i}}\right|=m
$$

и, следовательно, для доказательства теоремы достаточно показать, что $m<s$. Во множестве $I_{1}$ найдется пара различных номеров $p$ и $q$, поскольку по условию теоремы $\left|I_{1}\right| \geqslant 2$. Пусть, далее, $h_{p}$ - вершина из максимальной клики $K_{p}$ и $h_{q}-$ вершина из максимальной клики $K_{q}$, причем $h_{p}$ несмежна с $h_{q}$ (такие вершины найдутся, поскольку клика $K_{p}$ не совпадает с кликой $K_{q}$ ). Из несмежности вершин $h_{p}$ и $h_{q}$ следуют два вывода:

1) ни одна из вершин $h_{p}$ и $h_{q}$ не лежит во множестве $\left\{a_{1}, \ldots, a_{m}\right\}$, поскольку по предыдушей лемме каждая из $a_{j}$ смежна со всеми вершинами клик $K_{p}$ и $K_{q}$;

2 ) в $G$ вершины $h_{p}$ и $h_{q}$ соединены гамильтоновой цепью (простой цепью, проходящей по всем остальным вершинам графа), поскольку $G$ - МНГ граф.

Гамильтонова цепь из $h_{p}$ в $h_{q}$ не содержит отрезков $a_{i} a_{j}$, т.е. не может иметь вид

$$
h_{p} \bar{t}_{1} a_{i} a_{j} \bar{t}_{2} h_{q}
$$

поскольку иначе в графе $G$ найдется гамильтонов цикл

$$
h_{p} \bar{t}_{1} a_{i} h_{q} \bar{t}_{2}^{-1} a_{j}
$$

что противоречит негамильтоновости графа $G$.

Таким образом, гамильтонова цепь, соединяющая $h_{p}$ и $h_{q}$, обязана иметь вид

$$
h_{p} \bar{t}_{1} a_{j_{1}} \bar{t}_{2} a_{j_{2}} \bar{t}_{3} \ldots \bar{t}_{m} a_{j_{m}} \bar{t}_{m+1} h_{q}
$$

причем цепи $\bar{t}_{i}, i=2, \ldots, m$, содержат хотя бы по одной вершине. Без ограничения обшности считаем, что $j_{l}=l, l=1, \ldots, m$. Тогда гамильтонова цепь из $h_{p}$ в $h_{q}$ примет вид

$$
h_{p} \bar{t}_{1} a_{1} \bar{t}_{2} a_{2} \bar{t}_{3} \ldots \bar{t}_{m} a_{m} \bar{t}_{m+1} h_{q} .
$$

Обозначим для $i=2, \ldots, m$ через $b_{i}$ последнюю вершину в цепи $\bar{t}_{i}$. Пусть, дополнительно, $b_{1}$ - последняя вершина в цепи $\bar{t}_{1}$, если эта цепь не пуста, и $b_{1}$ совпадает с $h_{p}$, если цепь $\bar{t}_{1}$ пуста. 
В этих обозначениях гамильтонова цепь вьглядит следующим образом:

$$
h_{p} \bar{t}_{1} b_{1} a_{1} \bar{t}_{2} b_{2} a_{2} \bar{t}_{3} \ldots \bar{t}_{m} b_{m} a_{m} \bar{t}_{m+1} h_{q}
$$

причем новые цепи $\bar{t}_{i}, i=1, \ldots, m+1$, могут быть пустьми.

По лемме 1 получаем: $\left\{b_{1}, \ldots, b_{m}\right\} \subset K_{i_{1}} \cup \cdots \cup K_{i_{s}}$. Предположим, что найдется такое $i \in 1, \ldots, m$, что $b_{i}$ принадлежит клике $K_{q}$. Тогда в $G$ существует гамильтонов цикл

$$
h_{p} \bar{t}_{1} b_{1} a_{1} \bar{t}_{2} \ldots \bar{t}_{i} b_{i} h_{q} \bar{t}_{m+1}^{-1} a_{m} b_{m} \bar{t}_{m}^{-1} \ldots \bar{t}_{i+1}^{-1} a_{i}
$$

что противоречит негамильтоновости графа $G$. Следовательно, никакая из вершин $b_{1}, \ldots, b_{m}$ не лежит в клике $K_{q}$.

Теперь предположим, что $m \geqslant s$. Тогда, поскольку все вершины $b_{1}, \ldots, b_{m}$ лежат в кликах $K_{i_{1}}, \ldots, K_{i_{s}}$ и никакая из этих вершин не лежит в клике $K_{q}$, которая является одной из клик $K_{i_{1}}, \ldots, K_{i_{s}}$, найдется пара вершин $b_{i}$ и $b_{j}$, лежашая в одной клике. Следовательно, $b_{i}$ и $b_{j}$ соединены ребром в графе $G$. Кроме того, $a_{i} \in M_{I}$ и $a_{j} \in M_{J}$, где $I, J \in\left\{I_{1}, \ldots, I_{t}\right\}$. Поскольку $I_{1} \subset I_{2} \subset \cdots \subset I_{t}$, то либо $I \subseteq J$, либо $J \subseteq I$. Также выполняется одно из двух неравенств: $i<j$ или $j<i$. Проведем доказательство для случая, когда $i<j$ и $J \subseteq I$; в остальных трех случаях доказательство проводится аналогично. По лемме 1 получаем, что если некоторая вершина $c$, отличная от $a_{i}$, смежна с $a_{j}$, то она смежна и с вершиной $a_{i}$. Следовательно, и первая вершина в цепи $\bar{t}_{i+1}$ и первая вершина в цепи $\bar{t}_{j+1}$ смежны с $a_{i}$. Но тогда можно указать гамильтонов цикл в графе $G$ :

$$
h_{p} \bar{t}_{1} b_{1} a_{1} \bar{t}_{2} \ldots \bar{t}_{i} b_{i} b_{j} \bar{t}_{j}^{-1} a_{j-1} b_{j-1} \bar{t}_{j-1}^{-1} \ldots \bar{t}_{i+1}^{-1} a_{i} \bar{t}_{j+1} b_{j+1} a_{j+1} \bar{t}_{j+2} \ldots \bar{t}_{m+1} h_{q} a_{j}
$$

что противоречит с определением МНГ графа. Таким образом, неравенство $m \geqslant s$ приводит к противоречию. Теорема 1 доказана.

Приведем в виде следствия один частный случай.

СлЕдСТВИЕ. Если $G$ - МНГ граф, то для любого $I \subseteq R$ maкого, ито $|I| \geqslant 2$, выполнено неравенство

$$
n_{I} \leqslant|I|-1
$$

Согласно доказанной теореме для МНГ графа значения $n_{I}$ ограничены сверху при $|I| \geqslant 2$. Очевидно также, что $n_{\varnothing}=0$. Но остается вопрос об ограничении сверху величины $n_{\{i\}}=\left|M_{\{i\}}\right|, i=1, \ldots, r$. Легко видеть, что во множество $M_{\{i\}}=K_{i} \backslash \bigcup_{j \neq i} K_{j}$ входят только собственные вершины максимальной клики $K_{i}$, т.е. те вершины, которые принадлежат клике $K_{i}$ и не принадлежат другим максимальньм кликам из набора $K_{1}, \ldots, K_{r}$. В предыдущем разделе показано, что изучение МНГ графов можно свести к изучению упрощенных МНГ графов. Но для упрощенных графов по определению все $n_{\{i\}} \leqslant 1, i=1, \ldots, r$. Таким образом, набор $n_{I}, I \subseteq R, I \neq \varnothing$, задаваемьй упрощенным 
МНГ графом, обязан удовлетворять системе линейных неравенств (СЛН), состоящей из трех подсистем:

$$
\begin{aligned}
& \left\{x_{I} \geqslant 0, \quad I \subseteq R, \quad I \neq \varnothing,\right. \\
& \left\{\sum_{i=1}^{t} x_{I_{i}} \leqslant\left|I_{t}\right|-1, \quad I_{1} \subsetneq I_{2} \subsetneq \cdots \subsetneq I_{t} \subseteq R, \quad\left|I_{1}\right| \geqslant 2, \quad t \in 1, \ldots, r-1,\right. \\
& \left\{x_{\{i\}} \leqslant 1, \quad i \in 1, \ldots, r .\right.
\end{aligned}
$$

Множеством неизвестных этой системы являются $\left\{x_{I}: I \subseteq R, I \neq \varnothing\right\}$. Упростим указанную систему неравенств, перейдя к эквивалентной.

УТВЕРЖДЕНИЕ 2. Если в СЛН, задаваемой неравенствами (1) и (2), из неравенств (2) оставить только неравенства

$$
\begin{aligned}
& \left\{\sum_{i=1}^{t} x_{I_{i}} \leqslant t, \quad I_{1} \subsetneq I_{2} \subsetneq \cdots \subsetneq I_{t} \subseteq R, \quad\left|I_{i}\right|=i+1,\right. \\
& i=1, \ldots, t, \quad t \in 1, \ldots, r-1,
\end{aligned}
$$

то получим СЛН, әквивалентную исходной.

Доказательство следует из того, что любая цепочка включений $I_{1} \subsetneq I_{2} \subsetneq \ldots \subsetneq I_{s}$ $\subseteq R,\left|J_{1}\right| \geqslant 2$, следует из некоторой цепочки включений $I_{1} \subsetneq I_{2} \subsetneq \cdots \subsetneq I_{t} \subseteq R$, $\left|I_{t}\right|=i+1, i=1, \ldots, t$, такой, что $\left\{J_{1}, \ldots, J_{s}\right\} \subset\left\{I_{1}, \ldots, I_{t}\right\}$.

Таким образом, СЛН, составленная из неравенств (1)-(3) эквивалентна своей подсистеме, состоящей из неравенств (1), (4), (3). Далее эту подсистему станем называть МНГ-системой. Из вьшесказанного следует утверждение.

УТВЕРЖДЕНИЕ 3. Пусть граф $G$ - упрощенный МНГ граф с $r$ максимальными кликами, объединение которых дает әраф $G$. Набор $n_{I}, I \subseteq R, I \neq \varnothing$, задаваемый графом $G$, является иелочисленным решением МНГ-системы, однозначно определяемой значением натурального параметра $r>1$.

3. Оценка порядков и числа упрощенных МНГ графов. Пусть $G$ - упрощенный МНГ граф с $r$ максимальными кликами, объединение которых дает граф $G$. Напомним, что порядок графа $G$ равен $\sum_{I \subseteq R, I \neq \varnothing} n_{I}$. Рассмотрим линейную функцию $\tau(x)$, где $x=\left\{x_{I}: I \subseteq R, I \neq \varnothing\right\}:$

$$
\tau(x)=\sum_{I \subseteq R, I \neq \varnothing} x_{I} .
$$

Пусть максимум этой линейной функции на множестве всех решений МНГ-системы равен $C(r)$. Тогда порядок графа $G$ не превьшает $C(r)$. Обозначим через $P(r)$ максимум порядков упрощенных МНГ графов, имеющих ровно $r$ максимальных клик. Ясно, что при любом натуральном $r>1 P(r) \leqslant C(r)$.

Далее вычислим точное значение величины $C(r)$. 
УТВЕРЖДЕНИЕ 4. Для любого натурального $r>1$ верно равенство

$$
C(r)=2^{r-1}+\frac{r-3}{2} \cdot\left(\begin{array}{c}
r \\
{[r / 2]}
\end{array}\right)+r
$$

ДоКАЗАТЕЛЬСТВо. При фиксированном $r$ множество неизвестньх МНГ-системы это множество $x=\left\{x_{I}: I \subseteq R, I \neq \varnothing\right\}$. Количество неизвестных равно $2^{r}-1$. Отметим, что неизвестные, участвующие в неравенствах (4), и неизвестные, участвующие в неравенствах (3), образуют непересекающиеся множества. Поэтому МНГ-систему можно разделить на две независимые СЛН:

$$
\begin{aligned}
& \left\{\begin{array}{l}
x_{\{i\}} \geqslant 0, i \in 1, \ldots, r, \\
x_{\{i\}} \leqslant 1, \quad i \in 1, \ldots, r,
\end{array}\right. \\
& \left\{\begin{array}{c}
x_{I} \geqslant 0, \quad I \subseteq R, \quad|I| \geqslant 2, \\
\sum_{i=1}^{t} x_{I_{i}} \leqslant t, \quad I_{1} \subset I_{2} \subset \cdots \subset I_{t} \subseteq R, \quad\left|I_{i}\right|=i+1, \\
i=1, \ldots, t, \quad t \in 1, \ldots, r-1 .
\end{array}\right.
\end{aligned}
$$

Пусть линейная функция $\sum_{i=1}^{r} x_{\{i\}}$ имеет максимум $C_{1}(r)$ на множестве всех решений СЛН (5); линейная функция $\sum_{I \subseteq R,|I| \geqslant 2} x_{I}$ имеет максимум $C_{2}(r)$ на множестве решений системы (6). Легко видеть, что $C(r)=C_{1}(r)+C_{2}(r)$. Кроме того, очевидно, что $C_{1}(r)=r$.

Остается доказать, что

$$
C_{2}(r)=2^{r-1}+\frac{r-3}{2} \cdot\left(\begin{array}{c}
r \\
{[r / 2]}
\end{array}\right) .
$$

Обозначим $A=\{I \subseteq R:|I| \geqslant 2\}$. Пусть $\tilde{y}=\left\{y_{I}, I \in A\right\}$-произвольноецелочисленное решение системы (6). Докажем, что тогда сушествует решение $\tilde{y}^{(1)}=\left\{y_{I}^{(1)}, I \in A\right\}$ такое, что при всех $I \in A: 2 \leqslant|I| \leqslant\left[\frac{r}{2}\right]-1 y_{I}^{(1)}=0$, а при $I \in A:\left[\frac{r}{2}\right]+1 \leqslant|I| \leqslant r$ $y_{I}^{(1)}=y_{I}$, и, кроме того, целевая функция $\sum_{I \in A} x_{I}$ для решения $\tilde{y}^{(1)}$ не меньше, чем для $\tilde{y}: \sum_{I \in A} y_{I} \leqslant \sum_{I \in A} y_{I}^{(1)}$. Пусть само решение $\tilde{y}$ не годится в качестве $\tilde{y}^{(1)}-$ это означает, что существует $i_{0}, 2 \leqslant i_{0} \leqslant\left[\frac{r}{2}\right]-1$, которое равно минимальному номеру из таких номеров $i$, что $\max \left\{y_{I}:|I|=i\right\}>0$. Далее обозначим

$$
\begin{gathered}
\max \left\{y_{I}:|I|=i_{0}\right\}=y_{0}>0, \quad X=\left\{I \in A:|I|=i_{0}, y_{I}=y_{0}\right\}, \\
Y=\left\{J \in A:|J|=i_{0}+1, \exists I \in X \text { такое, что } I \subset J\right\} .
\end{gathered}
$$

Положим

$$
y_{I}^{\prime}=\left\{\begin{array}{lll}
y_{I}-1, & \text { если } I \in X, \\
y_{I}+1, & \text { если } I \in Y, \\
y_{I}, & \text { если } I \in A \backslash(X \cup Y) .
\end{array}\right.
$$

Очевидно, что $\tilde{y}^{\prime}=\left\{y_{I}^{\prime}: I \in A\right\}$-новое решение (6). По принципу Дирихле $|Y| \geqslant|X|$, поэтому целевая функция для нового решения не уменьшится: $\sum_{I \in A} y_{I} \leqslant \sum_{I \in A} y_{I}^{\prime}$. 
Теперь, если решение $\tilde{y}^{\prime}$ не годится в качестве $\tilde{y}^{(1)}$, то применим к $\tilde{y}^{\prime}$ те же самые рассуждения и получим решение $\tilde{y}^{\prime \prime}$. За конечное число таких шагов, не уменьшая целевой функции, получим искомое решение $\tilde{y}^{(1)}$.

Совершенно аналогично от решения $\tilde{y}^{(1)}$ можно перейти к решению $\tilde{y}^{(2)}$, в котором при всех $I \in A: 2 \leqslant|I| \leqslant\left[\frac{r}{2}\right]-1 y_{I}^{(2)}=0$, а при $I \in A:\left[\frac{r}{2}\right]+1 \leqslant|I| \leqslant r y_{I}^{(1)}=1$, и целевая функция $\sum_{I \in A} x_{I}$ для решения $\tilde{y}^{(2)}$ не меньше, чем для $\tilde{y}^{(1)}$. Для этого рассматривается $i_{0}:\left[\frac{r}{2}\right]+1 \leqslant i_{0} \leqslant r$ - максимальньй номер такой, что

$$
\begin{gathered}
\max \left\{y_{I}^{(1)}:|I|=i_{0}\right\}=y_{0}>1, \quad X=\left\{I \in A:|I|=i_{0}, y_{I}=y_{0}\right\} \quad \text { и } \\
Y=\left\{J \in A:|J|=i_{0}-1, \exists I \in X \quad \text { такое, что } I \supset J\right\} .
\end{gathered}
$$

Наконец от решения $\tilde{y}^{(2)}$ можно перейти к решению $\tilde{y}^{(3)}$ :

$$
y_{I}^{(3)}= \begin{cases}0, & \text { если } 2 \leqslant|I| \leqslant\left[\frac{r}{2}\right], \\ {\left[\frac{r}{2}\right]-1,} & \text { если }|I|=\left[\frac{r}{2}\right], \\ 1, & \text { если }\left[\frac{r}{2}\right]+1 \leqslant|I| \leqslant r .\end{cases}
$$

Мы исходили из произвольного решения $\tilde{y}$ и, поскольку на всех изложенных этапах целевая функция не уменьшалась, для решения $\tilde{y}^{(3)}$ целевая сумма самая большая, т.е. $C_{2}(r)=\sum_{I \in A} y_{I}^{(3)}$. Остается заметить, что

$$
\sum_{I \in A} y_{I}^{(3)}=2^{r-1}+\frac{r-3}{2} \cdot\left(\begin{array}{c}
r \\
{[r / 2]}
\end{array}\right)
$$

Утверждение 4 доказано.

Применение формулы Стирлинга предоставляет асимптотическое выражение для $C(r):$ при $r \rightarrow \infty$ имеем

$$
C(r)=2^{r} \cdot \sqrt{\frac{r}{2 \pi}} \cdot(1+o(1)) .
$$

Полученные результаты позволяют нам оценить сверху число $D(r)$ упрощенных МНГ графов, представимых в виде объединения $r$ своих максимальных клик.

СлЕДСТВИЕ. Для любого натурального $r>1$ верно неравенство

$$
D(r) \leqslant\left(\begin{array}{c}
C(r)+2^{r}-1 \\
C(r)
\end{array}\right)
$$

в частности,

$$
\lim _{r \rightarrow \infty} \frac{\log _{2} D(r)}{C(r)} \leqslant 1 .
$$


ДокАЗАТЕльство. Пусть даны графы $G$ и $G^{\prime}$ и в каждом из этих графов задан набор из $r$ максимальных клик, объединение которых дает весь граф. Тогда для графа $G$ строится набор $n_{I}, I \subset R, I \neq \varnothing$, и для графа $G^{\prime}$ строится набор $n_{I}^{\prime}, I \subseteq R, I \neq \varnothing$. Если графы $G$ и $G^{\prime}$ различны, то и наборы $n_{I}$ и $n_{I}^{\prime}$ должны быть различны. Таким образом, получаем, что количество упрощенных МНГ графов с фиксированным числом максимальных клик, объединение которых дает весь граф, конечно. Это количество ограничено сверху числом целочисленных решений МНГ-системы. Из утверждения 4 получаем, что для любого целочисленного решения $x_{I}^{\prime}, I \subseteq R, I \neq \varnothing$, МНГ-системы верно неравенство

$$
\sum_{I \subseteq R, I \neq \varnothing} x_{I}^{\prime} \leqslant C(r) .
$$

В сумме (8) $2^{r}-1$ целочисленных слагаемых $x_{I}^{\prime} \geqslant 0$. Число решений $L(r)$ неравенства (8) оценивает сверху число решений МНГ-системы, а следовательно, и значение $D(r)$. Нетрудно убедиться, что

$$
L(r)=\left(\begin{array}{c}
C(r)+2^{r}-1 \\
C(r)
\end{array}\right)
$$

(см., например, [5]).

Очевидно, что $L(r) \leqslant 2^{C(r)+2^{r}-1}$, поэтому $\log _{2}(L(r)) \leqslant C(r)+2^{r}-1$, откуда имеем (7). Следствие доказано.

В заключение отметим, что для любого натурального $r>1$ существует упрощенный МНГ граф, представляемый в виде объединения $r$ своих максимальных клик, и не представляемьй в виде объединения меньшего, чем $r$, числа максимальных клик. Этот граф можно задать через значения $n_{I}, I \subseteq R, R=\{1, \ldots, r\}: n_{R}=r-1, n_{I}=0$ при $2 \leqslant|I| \leqslant r-1, n_{\{i\}}=1$ для всех $i \in 1, \ldots, r$.

\section{СПИСОК ЦИТИРОВАННОЙ ЛИТЕРАТУРЫ}

[1] Емеличев В.А., Мельников О. И., Сарванов В. И., Тышкевич Р. И. Лекции по теории графов. М.: Наука, 1990.

[2] Ролдугин П. В. Максимально негамильоновые графы // Третий Всероссийский симпозиум по прикладной и промьшленной математике. Тезисы докладов. М.: ТВП, 2002. С. 238-239.

[3] Bondy J. A. Variations on the Hamiltonian theme // Canad. Math. Bull. 1972. V. 14. № 1. P. 57-62.

[4] Харари Ф. Теория граф̆ов. М.: Мир, 1973.

[5] Сачков В. Н. Введение в комбинаторные методы дискретной математики. М.: Наука, 1982.

Московский институт радиотехники, электроники и автоматики (МИРЭА)

E-mail: PavRoldugin@rambler.ru

Поступило

06.02.2003

Исправленный вариант

18.04.2003 